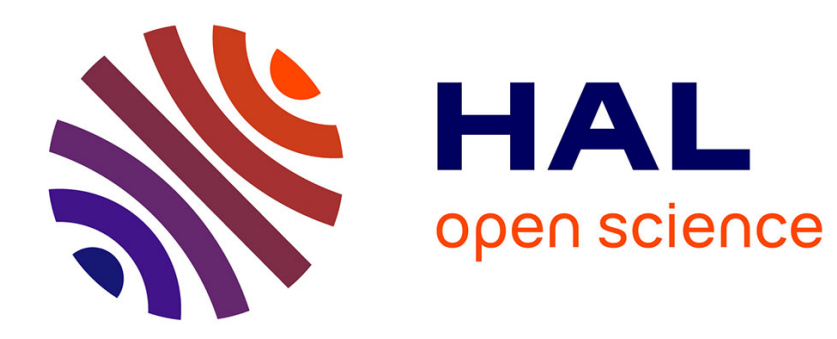

\title{
La spécialisation résidentielle dans le Sillon Lorrain
}

Julien Gingembre

\section{To cite this version:}

Julien Gingembre. La spécialisation résidentielle dans le Sillon Lorrain. Revue Géographique de l'Est, 2016, 56 (3-4), 10.4000/rge.5922 . hal-02941851

\section{HAL Id: hal-02941851 \\ https://hal.science/hal-02941851}

Submitted on 17 Sep 2020

HAL is a multi-disciplinary open access archive for the deposit and dissemination of scientific research documents, whether they are published or not. The documents may come from teaching and research institutions in France or abroad, or from public or private research centers.
L'archive ouverte pluridisciplinaire HAL, est destinée au dépôt et à la diffusion de documents scientifiques de niveau recherche, publiés ou non, émanant des établissements d'enseignement et de recherche français ou étrangers, des laboratoires publics ou privés. 


\section{La spécialisation résidentielle dans le Sillon Lorrain}

Residential specialization in the Sillon Lorrain

Räumliche Spezialisierung im Sillon Lorrain

\section{Julien Gingembre}

\section{(2) OpenEdition}

\section{Journals}

Electronic version

URL: http://journals.openedition.org/rge/5922

ISSN: 2108-6478

\section{Publisher}

Association des géographes de l'Est

\section{Printed version}

Date of publication: 1 December 2016

ISSN: 0035-3213

\section{Electronic reference}

Julien Gingembre, «La spécialisation résidentielle dans le Sillon Lorrain », Revue Géographique de l'Est [Online], vol. 56 / n³-4 | 2016, Online since 29 November 2016, connection on 08 September 2020. URL : http://journals.openedition.org/rge/5922

This text was automatically generated on 8 September 2020

Tous droits réservés 


\title{
La spécialisation résidentielle dans le Sillon Lorrain
}

\author{
Residential specialization in the Sillon Lorrain \\ Räumliche Spezialisierung im Sillon Lorrain
}

Julien Gingembre

1 En deux décennies, la métropolisation s'est imposée comme un concept central pour établir une nouvelle lisibilité du fait urbain (Ghorra-Gobin, 2010 et 2015 ; Halbert, 2010). Depuis les années 1980, l'observation de ces répercussions spatiales et du ralentissement des rythmes d'urbanisation dans les pays développés a mis au jour les processus nouveaux qui transforment de facto l'urbain. En effet, la concentration des flux (matériels, immatériels, humains) et des fonctions rares dans les grandes villes s'accompagne d'une urbanisation diffuse, parfois soumise à des fractures (Leroy, 2000). La structure et les dynamiques internes des agglomérations sont considérablement modifiées. Celles-ci s'écartent de la notion d'urbs, de cité, conséquence d'une périurbanisation étalée, en nappes mais aussi en archipels. Aujourd'hui, plus de $95 \%$ des français résident sous l'influence d'une ville (Brutel et Levy, 2011). Les recompositions territoriales en œuvre ont aussi pour conséquence de modifier l'organisation sociospatiale des villes (Lacour et Puissant, 1999). La diffusion de l'urbanité au delà des frontières traditionnelles de la ville conduit à l' " exportation " des dysfonctionnements métropolitains. La manifestation des phénomènes ségrégatifs n'est donc plus seulement circonscrite à la ville-centre, mais s'étend jusqu'aux communes les plus éloignées des aires urbaines.

2 Par leur diffusion, ces phénomènes ségrégatifs sont devenus une caractéristique importante des aires urbaines formées de mosaïques d'espaces socialement fragmentés. Cette fragmentation spatiale s'accompagne d'une ségrégation sociale qui peut se définir comme «un ensemble de processus, de comportements, de procédures qui, volontairement ou non, permettent et produisent des inégalités socio-spatiales concentrées en certains lieux » (Gaschet et Lacour, 2008). Ces inégalités constituent des barrières à l'intégration et des freins à l'accessibilité réelle. Elles pénalisent ou rendent difficile l'insertion et favorisent ou accélèrent l'éviction ou l'exclusion (ibid.). À la fois 
situation et processus, la ségrégation est traduite à l'échelle locale par une concentration spatiale des populations, et par une segmentation des territoires en sous-espaces de repli. À l'échelle des aires urbaines, les résultats hérités de ces processus changent d'échelle et s'inscrivent différemment dans la compréhension du tissu urbain. Les nécessités du fonctionnement métropolitain (centralité des marchés d'emplois, mutualisation des services rares, localisation des grands équipements, concentration des infrastructures de transport) posent différemment la question de la cohésion territoriale des aires urbaines. Ces processus, d'abord observés dans les plus grands ensembles urbains, n'échappent pas aux métropoles que l'on qualifie d' " intermédiaires ». Cette désignation concerne les villes appartenant à l'armature métropolitaine française mais qui ne présentent pas un rayonnement international suffisamment important : généralement, la fourchette 200000 à 500000 habitants dans l'aire urbaine est utilisée (Dumont, 2006).

3 Si la ségrégation urbaine est un phénomène très ancien, la métropolisation a conduit à lui accorder une attention renouvelée. Ainsi, les chercheurs ont dû adapter leurs échelles d'analyse à l'ampleur spatiale prise par ce processus. Pour cela, l'utilisation d'indices de ségrégation spatiaux permet « de qualifier et de confronter la répartition dans l'espace des différents groupes, de comparer les situations entre différentes villes ou encore de réaliser des analyses diachroniques "(Apparicio, 2000). Enfin, la thématique s'est imposée au cours des années 2000 comme une préoccupation majeure dénoncée par exemple par la loi relative à la solidarité et au renouvellement urbain (SRU), avec la volonté de questionner les aspects sociaux et de mesurer les externalités négatives causées par la métropolisation.

4 Les débats autour de la ségrégation urbaine et de la métropolisation sont vifs. L'ambition de cet article est de porter leur analyse à l'échelle d'un réseau de villes, ce qui amène encore à se poser plus de questions. En effet, si P. Veltz a montré que les relations horizontales entre les grandes agglomérations forment de nouveaux « territoires-réseaux » (Veltz, 2005), les relations héritées, tissées entre les villes et leurs territoires proches, demeurent fortes, bien qu'elles aient dépassé la traditionnelle distinction ville-campagne. Pour l'illustrer, cette recherche est appliquée à l'ancienne région Lorraine ${ }^{1}$, et plus particulièrement à son axe métropolitain, le Sillon Lorrain, où la question de la métropolisation est posée avec une acuité particulière compte-tenu du polycentrisme structurel hérité de l'histoire.

5 Le travail qui est proposé porte sur les indices de spécialisation résidentielle. Cela constitue une démarche pertinente pour mettre en évidence les structures spatiales qui organisent le territoire. Dans un premier temps, l'article présente le cadre géographique de l'ancienne région Lorraine et de son axe urbain du Sillon Lorrain. Dans un second temps sera défini le cadre méthodologique portant sur les quotients de localisation. Puis, la démonstration s'intéresse au contraste entre plusieurs populations : les cadres, les chômeurs et les employés-ouvriers.

\section{La Lorraine : une bicéphalie en voie de structuration politique}

6 L'axe métropolitain lorrain court de Nancy à Luxembourg et épouse le cours de la Moselle (Paulet, 2010) (figure 1). Il relève de flux méridiens parfois qualifiés de " lotharingiens » (Frécaut, 1983 ; Auburtin, 2014). L'histoire de ce territoire fut traversée 
par plusieurs grandes secousses. On retiendra la partition de 1871, les deux guerres mondiales, mais aussi des phases de prospérité et encore la fonction occupée par ce territoire au début de l'aventure européenne.

Figure 1 : éléments de la géographie lorraine

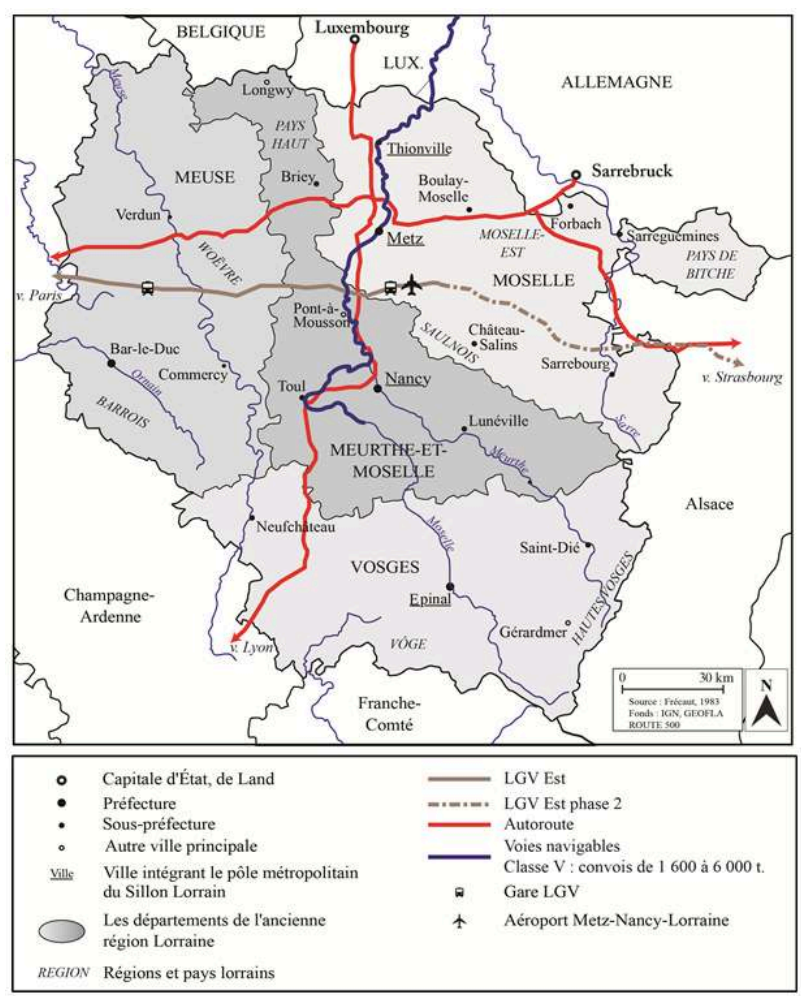

7 Au sein de ce couloir, Nancy et Metz sont deux villes économiquement et démographiquement similaires mais aux caractères métropolitains incomplets. Thionville, au Nord, et Épinal, au Sud, étirent cet axe urbain, aux échanges multiples (flux domicile-travail et domicile-étude, déplacements liés aux loisirs et à la consommation). Les franges septentrionales du territoire sont sous l'influence des métropoles frontalières de Luxembourg et, secondairement, de Sarrebruck (figure 2). Les aires urbaines des quatre villes s'étendent du Nord au Sud du territoire lorrain. Celui-ci s'organise également avec des espaces ruraux faiblement peuplés (la plaine de la Woëvre en Meuse, la Vôge au sud d'Épinal, ou encore le Saulnois au nord-est de Nancy). La Lorraine présente des indicateurs démographiques et économiques d'un dynamisme modeste. Entre 2006 et 2011, l'accroissement de la population atteint 0,64 $\%$, soit un peu moins de 15000 habitants, la Lorraine devançant seulement la Champagne-Ardenne et le Nord-Pas-de-Calais dans le classement des anciennes régions françaises (INSEE, populations légales 2011). 
Figure 2 : localisation des emplois frontaliers dans les communes du nord-lorrain (2010)
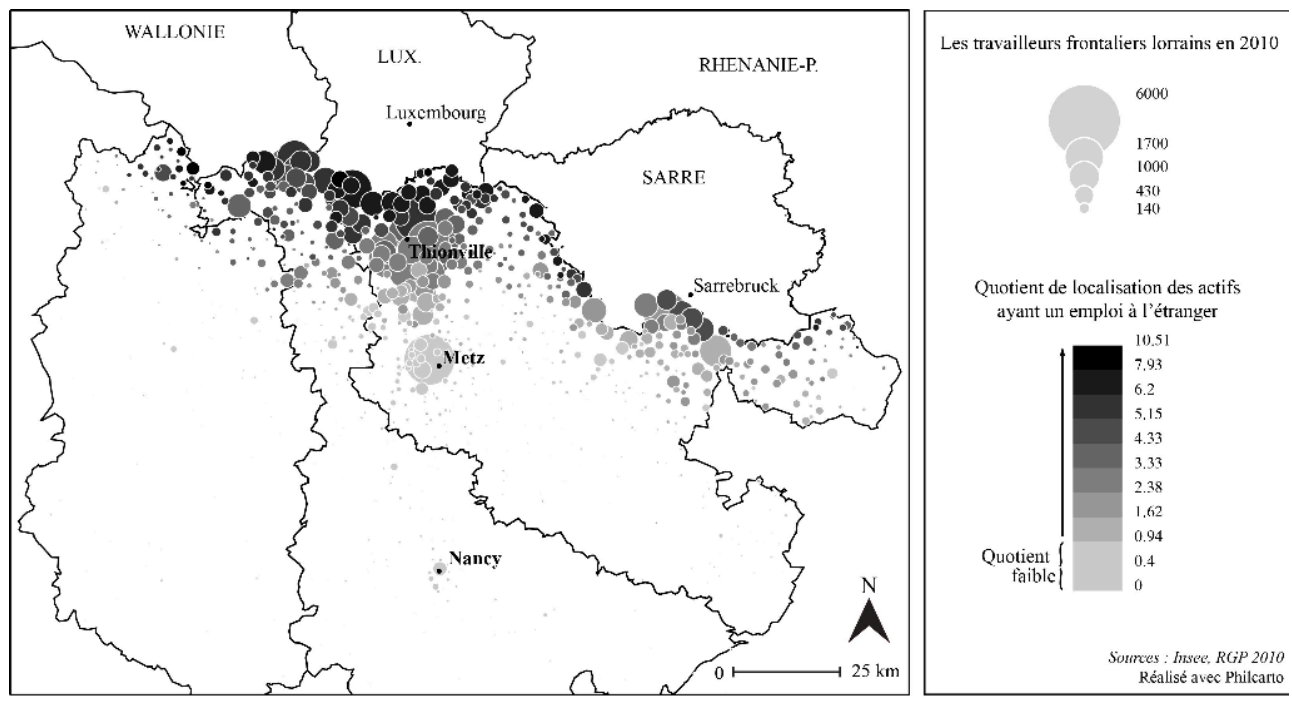

L'émergence de territoires vécus dépassant le cadre des aires urbaines a conduit les élus des quatre principales agglomérations lorraines (du nord au sud : Thionville, Metz, Nancy et Épinal) à promouvoir un rapprochement. Ce couloir métropolitain est ainsi devenu une réalité politique avec la démarche du Sillon Lorrain (Gingembre et Husson, 2014). Le rapprochement actif et endogène des villes de Metz et Nancy est engagé au début des années 1990. Cela constitue une réminiscence d'une stratégie plus ancienne, mais venue de l'État, celle de la métropole d'équilibre Nancy-Metz-Thionville. Imposée à partir de 1966, cette démarche avait tenté une union stratégique autour de cette situation de proximité, considérée comme un avantage, dans l'optique de constituer une conurbation linéaire, structurée autour des projets logistiques de l'autoroute A31, du TER (Transport Express Régional) et de la Moselle canalisée. L'échec relatif de la stratégie à l'échelle nationale et le développement concurrentiel des deux villes lorraines ont rapidement enterré l'ambition métropolitaine.

Dans le contexte des années 1990 (mondialisation affirmée, effondrement du bloc de l'Est, construction européenne, poursuite de la décentralisation et, localement, désindustrialisation et démilitarisation de la Lorraine), l'idée d'une métropole multipolaire s'impose progressivement avec la reconnaissance d'un contrat métropolitain dans le Contrat de plan État-Région de troisième génération (1994-1999). Épinal et Thionville se joindront ensuite à cette initiative portée par la création de l'association du Sillon Lorrain en 2005 comme opération soutenue par la DIACT ${ }^{2}$.

Cette volonté métropolitaine forte et conduite sur plus d'une décennie par les élus des villes du Sillon Lorrain a entrainé le législateur à intégrer dans la Réforme des collectivités territoriales de 2010 le volet " pôle métropolitain " 3 , parallèlement à celui des «métropoles » (Béhar et al., 2011). Ces actions se concrétiseront avec la création du pôle métropolitain du Sillon Lorrain en décembre 2011. Il s'agit de la première expérience conduite en France. Le Sillon Lorrain associe les intercommunalités de Thionville, Metz, Nancy et Épinal, et comptait ainsi 632016 habitants en 2011, soit plus d'un quart de la population lorraine (INSEE, populations légales 2011).

11 Le pôle métropolitain s'impose comme l'organe permettant aux quatre villes de définir une orientation stratégique à l'échelon métropolitain. Il représente également une échelle de coopération permettant aux quatre agglomérations de constituer un 
interlocuteur unique sur la scène française et européenne, notamment dans le cadre de la Grande Région ${ }^{4}$ ou de la nouvelle région Grand Est.

12 Le Sillon Lorrain relève d'une configuration particulière, assez atypique, liée à la présence dans un secteur relativement restreint de deux villes de taille équivalente qui concentrent une part importante du tissu productif lorrain. Cela génère des flux domicile-travail qui jouent un rôle structurant pour le pôle métropolitain (Serre, 2013). De même, la Lorraine, qui peut être déclinée en modèle de région frontalière française (figure 2), est marquée par la progression du travail frontalier. Celui-ci est très polarisé et asymétrique, car principalement orienté vers le Luxembourg (en 2011, 9,7 \% des actifs Lorrains travaillaient à l'étranger, contre 8,6 \% en 2006).

(icularisme de l'armature urbaine lorraine nous amène à déployer une démarche déjà expérimentée ailleurs (Fusco et Scarella, 2011 ; Diop, 2011). L'objectif est de faire ressortir les grandes sectorisations de la spécialisation résidentielle en Lorraine afin d'en permettre une lecture globale. Les mécanismes sociaux qui sous-tendent aux phénomènes de spécialisation ne seront pas étudiés ici, mais des hypothèses seront avancées.

14 L'application de la mesure de la spécialisation résidentielle à la Lorraine pose plusieurs interrogations. La première concerne le caractère bicéphale de l'ancienne région: les deux pôles de Nancy et Metz, distants d'à peine plus de 60 kilomètres, ont connu des trajectoires différentes que le rapprochement opéré dans les années 1990 n'a pas effacé. Metz se tourne maintenant vers le pôle luxembourgeois (avec lequel elle intègre la "Quattropole » avec Sarrebruck et Trèves, association de coopération transfrontalière), tandis que Nancy continue de se poser comme un centre régional de services, notamment dans le domaine universitaire. Les deux villes sont des pôles plus tertiaires qu'industriels. Ils concentrent les emplois qualifiés, notamment les fonctions métropolitaines supérieures (Calzada et Cocher, 2009), mais montrent des signes de métropolisation incomplète. La similarité entre Nancy et Metz pourrait générer des organisations sociospatiales semblables. Parallèlement, la question des schémas observables dans les espaces à l'écart des dynamiques métropolitaines propres au Sillon Lorrain se pose avec une acuité particulière. C'est notamment le cas du pays d'Épinal, en amont de la vallée de la Moselle et situé à l'opposé des franges frontalières les plus vives: ce secteur constitue un enjeu important pour le pôle métropolitain dans l'optique d'équilibrer les forces et d'ouvrir la région vers la vallée du Rhône.

15 En Lorraine, le legs industriel est encore considérable, particulièrement en Moselle, où les difficultés dans la sidérurgie ont entrainé de récentes suppressions d'emplois. Les secteurs concernés (vallées de l'Orne et de la Fensch), encore très industrialisés, montrent des signes de reprise démographique rendue possible par l'attractivité luxembourgeoise (Piralla, 2014). Cela nous amène à s'interroger sur les types de spécialisation provoqués par l'attractivité luxembourgeoise: quelles spécificités socioéconomiques sont générées?

16 Pour évaluer ces hypothèses de départ, nous reviendrons sur la méthodologie qui est appliquée avant d'en présenter les résultats. 


\section{Cadre méthodologique pour mesurer les schémas résidentiels}

17 Les interactions entre des processus sociaux et les espaces dont ils sont le support produisent une spécialisation résidentielle plus ou moins forte. Afin de la mesurer, il faut donc déterminer dans un premier temps quels groupes sociaux étudier et, dans un second temps, définir quelle maille spatiale mobiliser.

18 Afin de retenir les groupes sociaux les plus représentatifs des disparités sociospatiales au niveau du territoire lorrain, et plus particulièrement au sein du Sillon, notre choix s'est porté sur la nomenclature des professions et catégories socioprofessionnelles (PCS) de l'INSEE. Cette nomenclature multicritère (métier, statut, qualification) offre des données statistiques disponibles à un niveau très fin et les recensements successifs autorisent les comparaisons dans le temps. Le premier niveau de cette nomenclature est composé de huit groupes socioprofessionnels. Parmi eux, nous en avons choisi trois. La première catégorie retenue est celle cadres et professions intellectuelles supérieures (CPIS, les cadres). Regroupant des professions qui appliquent des connaissances approfondies dans le domaine scientifique ainsi que des cadres qui ont des responsabilités importantes dans la gestion des entreprises, cette catégorie s'impose comme un des moteurs de la métropolisation (Julien, 2002). Les deux autres catégories choisies sont celles des employés et des ouvriers, deux groupes qui sont ici rassemblés. En effet, la frontière entre ces deux groupes tissés par de nombreuses interdépendances n'est pas facile à tracer: au sein de la catégorie des ouvriers, en dehors du noyau dur constitué par les ouvriers de production qualifiés de la grande industrie, les ouvriers non qualifiés se rapprochent de certaines professions d'employés. Ces deux groupes forment la masse principale de la population active et sont caractérisés par de fortes similitudes en termes de niveau de vie et de localisation des emplois (Fusco et Scarella, 2011). Enfin, nous avons parallèlement sélectionné les données de l'INSEE des populations de chômeurs, groupe retenu pour sa fragilité socioéconomique, en opposition notamment avec la catégorie des cadres. individus (statut social, mode de vie). Ces différentes catégories se différencient par leur budget logement, leurs loisirs, le type d'aménités recherchées, ou encore le degré plus ou moins contraint du choix de mobilité et de localisation résidentielle. Les données sont issues du recensement de 2011 et concernent les actifs occupés âgés de 15 à 64 ans et les chômeurs au lieu de résidence. Les données du recensement de 1999 ont également été utilisées afin d'illustrer les évolutions.

Le quotient de localisation sert ici d'indice de spécialisation. Il permet de mesurer la concentration relative d'un groupe au sein d'un ensemble plus grand. Un quotient inférieur à 1 correspond à une population-cible sous-représentée. Supérieur à 1 , le quotient indique une population-cible surreprésentée. Enfin, lorsqu'il est proche de 1, la population-cible est représentée de manière égale à l'ensemble de l'aire d'étude (Apparicio, 2000).

Le calcul du quotient de localisation: $Q L i=\frac{(\mathrm{xi} / \mathrm{ti})}{(\mathrm{x} / \mathrm{T})}$

$21 \mathrm{xi}=$ population du groupe $\mathrm{x}$ résidant dans l'unité spatiale i (le canton ou la commune)

$22 \mathrm{X}=$ population du groupe $\mathrm{x}$ résidant dans l'aire d'étude (la Lorraine) 

une catégorie motrice des dynamiques métropolitaines, ce qui a notamment été révélé par les travaux sur les fonctions métropolitaines de l'INSEE (Julien, 2002 ; Van Puymbroeck et Reynard, 2010), les chômeurs sont une population vulnérable qui peuvent subir les externalités négatives des métropoles. Les employés-ouvriers constituent quant à eux la majeure partie de la population active et présentent donc une répartition homogène sur le territoire.

\section{A. Les cadres fortement concentrés dans le Sillon Lorrain} proche périphérie, ce qui dessine bien l'axe métropolitain lorrain (figure 3). Cette présence est le résultat de la répartition des fonctions économiques et des besoins spécifiques du marché de l'emploi des grandes villes. Les cadres travaillent majoritairement dans les agglomérations centrales des grandes aires urbaines, et occupent des emplois bien rémunérés. Leurs ressources financières leur permettent de supporter le surcoût d'une installation dans les espaces centraux ou les marges périurbaines immédiates (Donzelot, 2004).

Les quotients les plus élevés se situent dans les cantons des pôles urbains de Metz et Nancy $^{5}$ et dans leur couronne plus ou moins immédiate, formant des auréoles périurbaines bien lisibles. À Metz et dans sa périphérie, les quotients sont très élevés (supérieurs à 1,5), tant au cœur de l'agglomération que dans les cantons périurbains des coteaux de Moselle (à l'ouest) et du Pays messin (à l'est). Concernant Nancy, si les cantons périphériques concentrent les cadres de manière moins forte (entre 1,1 et 1,5), les secteurs centraux de l'agglomération montrent les quotients les plus élevés en

Revue Géographique de l'Est, vol. 56 / n³-4 | 2016 
Lorraine : les cantons nancéiens ont des quotients supérieurs à 2,3. À la mesure de l'éloignement des ces deux agglomérations, la concentration des cadres se fait de moins en moins élevée.

Figure 3 : localisation des cadres et professions intellectuelles supérieures dans les cantons lorrains (2011)

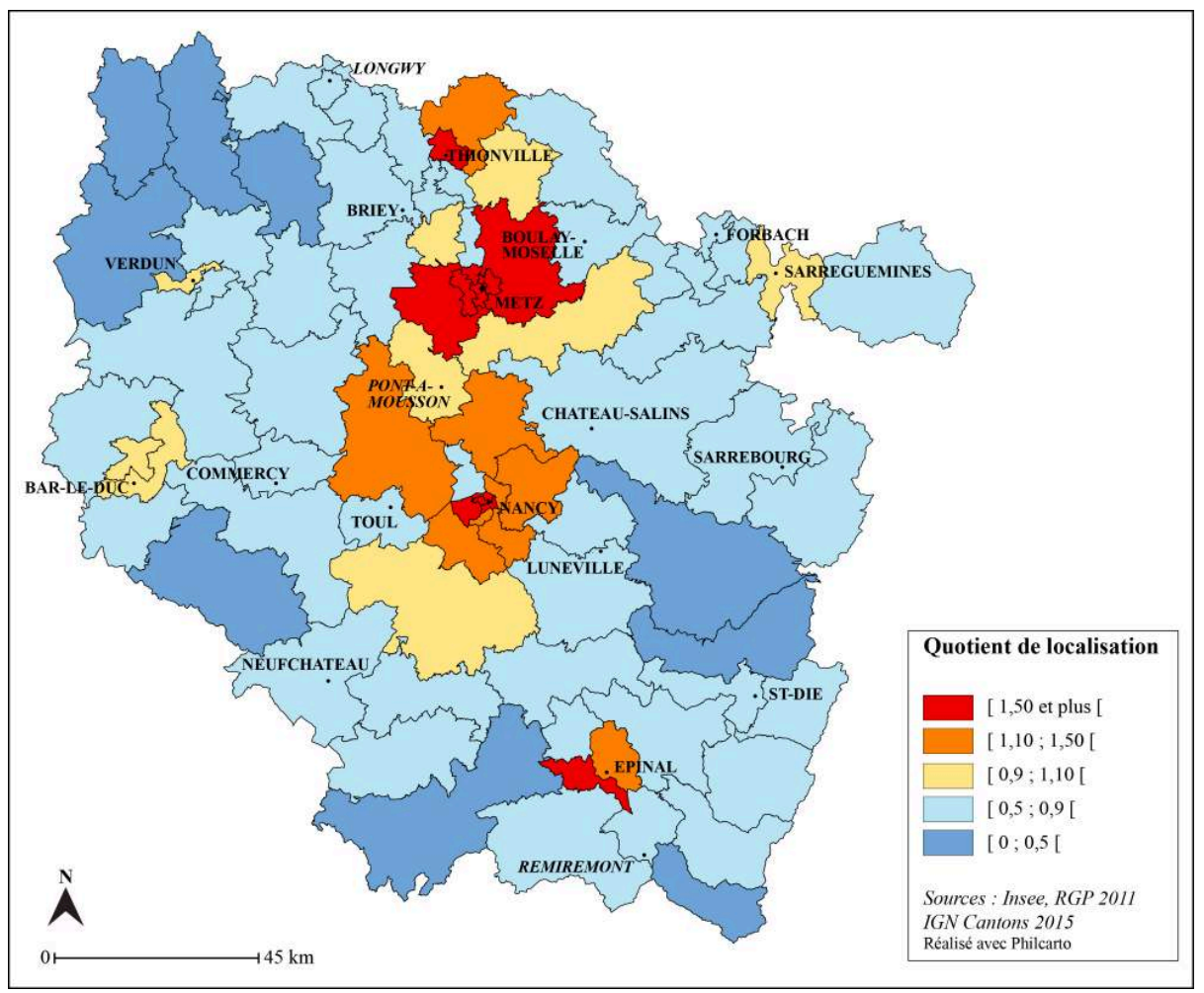

La carte dessine, en dehors du bicéphalisme Nancy-Metz, deux autres secteurs de concentration. Le premier correspond à la zone frontalière au nord du Sillon Lorrain. Elle réunit deux cantons qui concentrent les cadres (Thionville et, plus au nord, Yutz), en lien avec le marché d'emploi luxembourgeois situé à une trentaine de minutes en voiture : par leur accessibilité, ces cantons sont ceux où les impacts sur le coût du foncier et de l'immobilier sont les plus forts. Cela confirme que Luxembourg-ville étire sa zone d'attractivité jusqu'à Thionville et Metz (Diop, 2011 ; figure 2). La seconde est composée du pôle urbain d'Épinal. La préfecture des Vosges dispose d'assez de rayonnement pour parvenir à polariser des populations de cadres. Ailleurs, les cadres se font plus rares et sont surtout présents dans les petits pôles urbains (Bar-le-Duc, Sarreguemines, Verdun). 
Figure 4 : localisation des cadres et professions intellectuelles supérieures dans les cantons lorrains (1999)

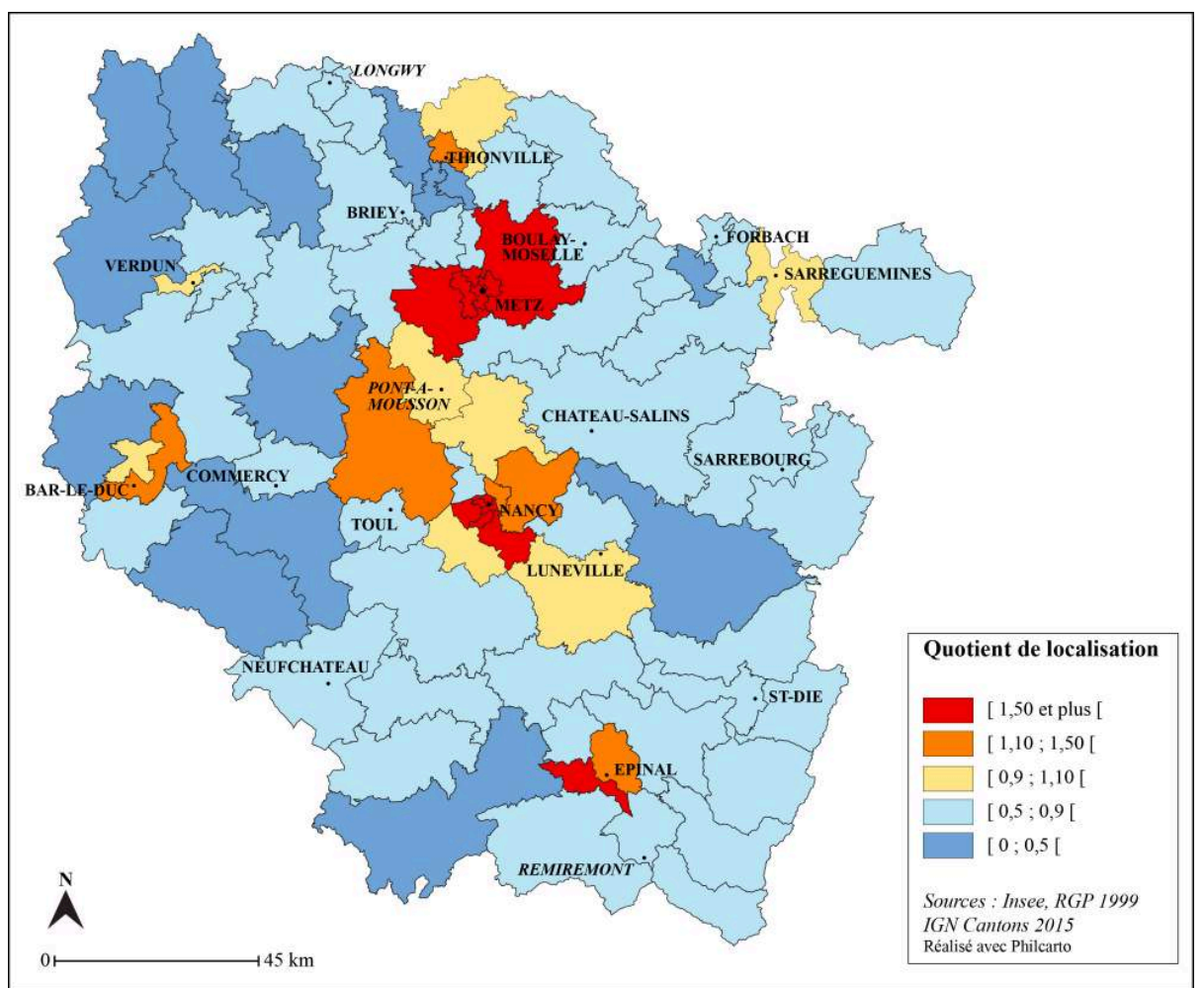

En matière d'évolution depuis 1999, la concentration résidentielle des cadres au sein de ces espaces suit les mêmes trajectoires. On observe d'abord une forte extension de l'espace de concentration des cadres à la frontière du Luxembourg, particulièrement dans le canton de Yutz (le plus septentrional du couloir mosellan) et en direction de Thionville, le long de l'axe de communication principal constitué par l'autoroute A31. L'attrait des petites communes périurbaines frontalières y joue un rôle central (Berrard et Debard, 2011). Inversement, les cantons du « Pays-Haut » (entre Briey et Longwy) n'affichent pas d'évolution positive des concentrations de cadres, le secteur ne semblant pas profiter de l'attractivité luxembourgeoise pour cette population qualifiée.

Plus généralement, l'augmentation du nombre de cadres est à l'origine d'une extension des espaces convoités par cette population autour des agglomérations de Metz et Nancy. Ce phénomène est notamment imputable à la progression de leur mobilité et à des choix résidentiels plus périurbains. À Nancy, les taux ont même diminué dans les cantons centraux, ce qui révèle une certaine dilution des quotients au sein de l'aire urbaine.

\section{B. Des chômeurs peu concentrés et en partie localisés en dehors du Sillon lorrain}

32 La localisation des chômeurs en Lorraine ne présente pas de poches de concentration aussi nettes que ce qui est dessiné pour la présence des cadres (figure 5). Ainsi, aucun canton ne présente des quotients très forts, c'est-à-dire supérieurs à 1,5.

La localisation résidentielle des chômeurs répond à deux logiques : d'une part, une forte présence dans les pôles urbains et les bassins industriels, ce qui s'observe de 
manière générale en France (Perrin et Rousier, 2002) ; d'autre part, une diffusion localisée sur les marges rurales de la Lorraine. Cette répartition constatée ici rejoint les observations réalisées par l'INSEE (Bischoff et Calzada, 2010).

Figure 5 : localisation des chômeurs dans les cantons lorrains (2011)

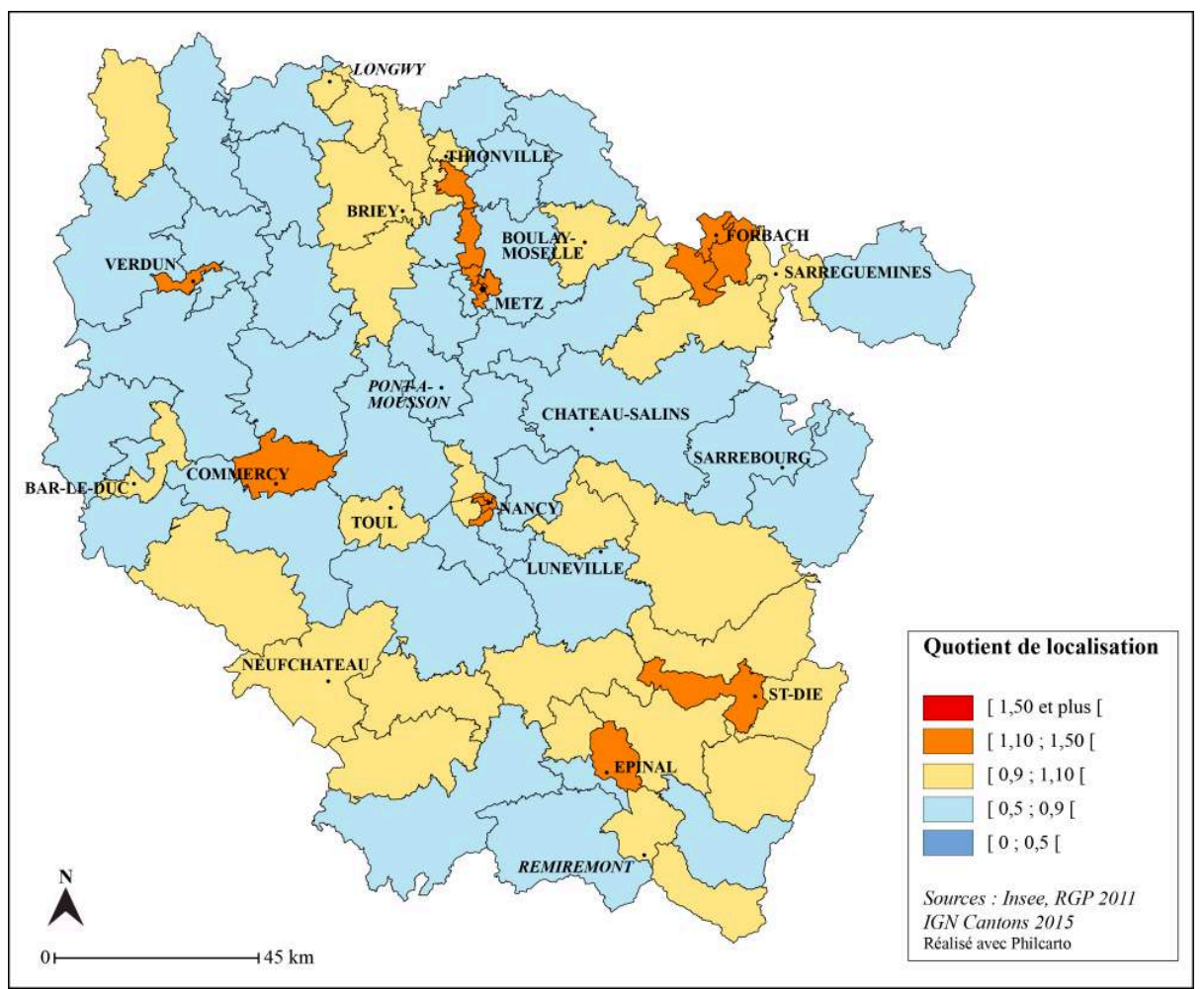

Comme dans les autres grands pôles urbains français, la concentration des chômeurs à Nancy et Metz est liée à la localisation des possibilités d'emploi, des structures d'aide au retour à l'emploi et des logements sociaux.

Dans le cas des bassins industriels lorrains, notamment dans la zone d'emploi de SaintDié6 et dans le bassin de vie de « Moselle-Est » (ancien bassin houiller de Forbach), cette surreprésentation est la conséquence d'un chômage endémique hérité des crises subies : dans les Vosges, mutation de l'industrie textile qui n'est plus une activité de maind'œuvre; en Moselle, fermeture des mines de charbon et déclin des industries qui les accompagnaient. Enfin, les cantons de la vallée de la Moselle entre Metz et Thionville combinent donc ces deux spécificités : une conurbation densément peuplée au cœur d'un bassin sidérurgique en difficulté.

Ailleurs, c'est surtout l'éloignement des grands pourvoyeurs d'emplois (les marchés d'emplois locaux sont faibles ou quasi inexistants) et des logiques résidentielles qui causent cette forte représentation des chômeurs. En effet, les centres urbains et les premières couronnes, jouissant d'une bonne accessibilité, sont, d'un point de vue financier, difficilement abordables pour les chômeurs. 
Figure 6 : localisation des chômeurs dans les cantons lorrains (1999)

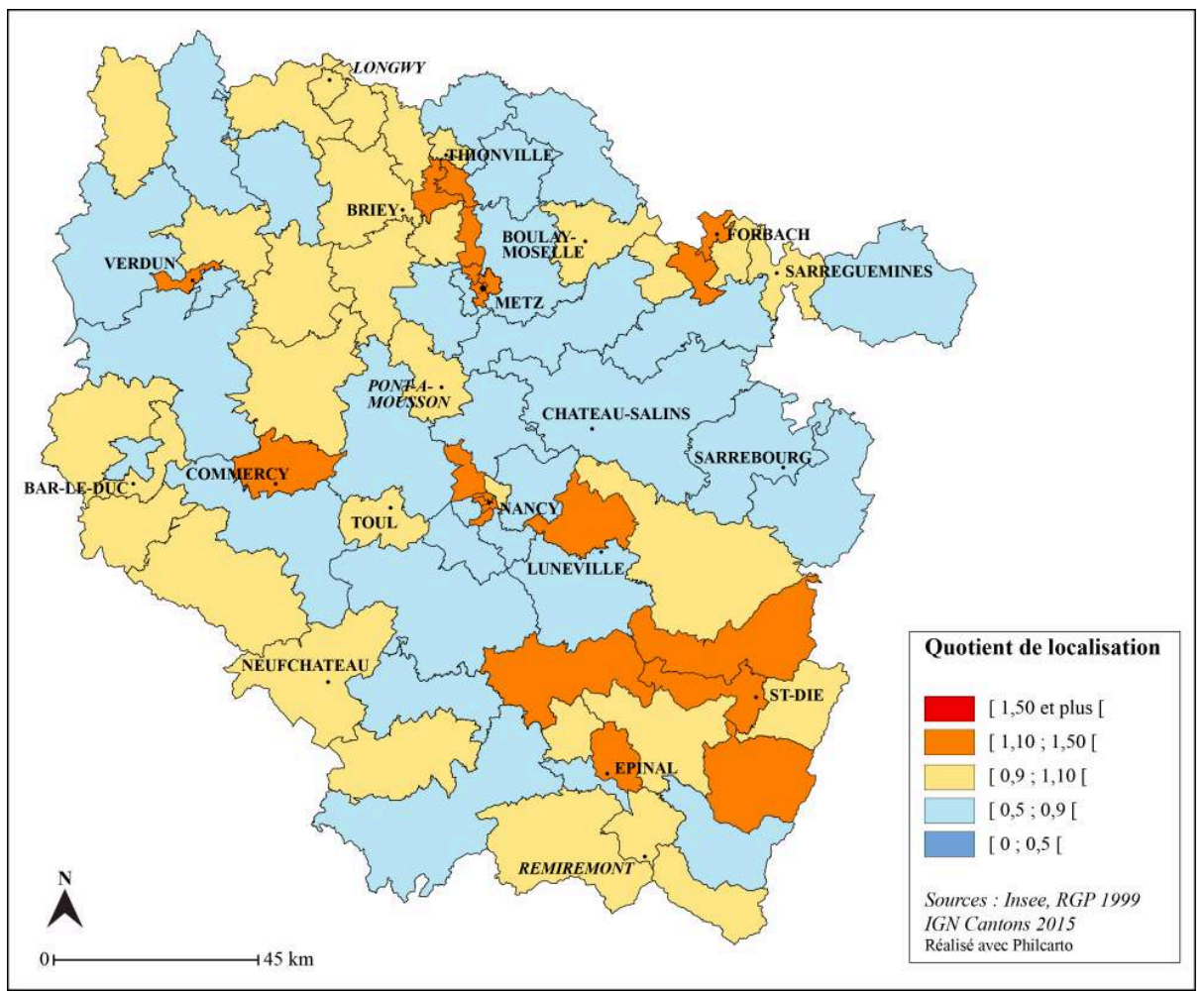

La localisation des chômeurs n'a pas connu de profonds bouleversements entre 1999 et 2009, mais les espaces de concentrations majeurs se sont rétractés durant cette décennie. C'est notamment le cas dans le nord-est des Vosges (autour de Saint-Dié), dans les cantons de « Moselle-Est » et dans le « Pays-Haut ».

Les figures 3 à 6 offrent une grande richesse de nuances mais l'échelon cantonal ne permet pas une lecture en détail. Pour un décryptage plus clair nous avons choisi de réduire l'éclairage à un exemple, l'unité urbaine de Nancy, en y mobilisant la maille communale (figure 7).

Le recoupement des deux cartes montre à la fois des superpositions et des spécificités visibles à cet échelon. Les cadres (carte de gauche) sont très localisés dans la communecentre de Nancy et dans les communes des coteaux et du plateau (à l'ouest et au nord) ayant préservé leurs aménités (Laxou, Villers) ou leur caractère villageois (Dommartemont, Eulmont). À l'inverse, les communes du nord-ouest de Nancy (Frouard, Pompey, à la confluence entre la Moselle et la Meurthe), encore en phase avec une désindustrialisation tardive, présentent des quotients moyens ou faibles.

Les chômeurs sont aussi localisés dans les communes centrales de l'agglomération nancéienne (carte de droite). Cette donnée traduit une certaine mixité des espaces urbains, les communes les plus grevées de chômeurs rassemblant les quartiers de logement social (Le Haut-du-Lièvre à cheval sur Laxou, Nancy et Maxéville, La Californie à Jarville, Les Nations à Vandoeuvre). Cette mixité, si elle existe bien à l'échelle de la commune, prend une tournure inverse à l'échelle des quartiers. Pour l'essentiel, ces sites sont en rénovation urbaine (Plan National de Rénovation Urbaine). Inversement, le déficit de logements sociaux pointé du doigt par la loi SRU concerne par exemple Villers et Bouxières. La spécialisation dans le résidentiel individuel s'accompagne ainsi d'une part moindre de chômeurs. 
Figure 7 : les disparités de localisation résidentielle des chômeurs et des CPIS au sein de l'agglomération de Nancy (2011)

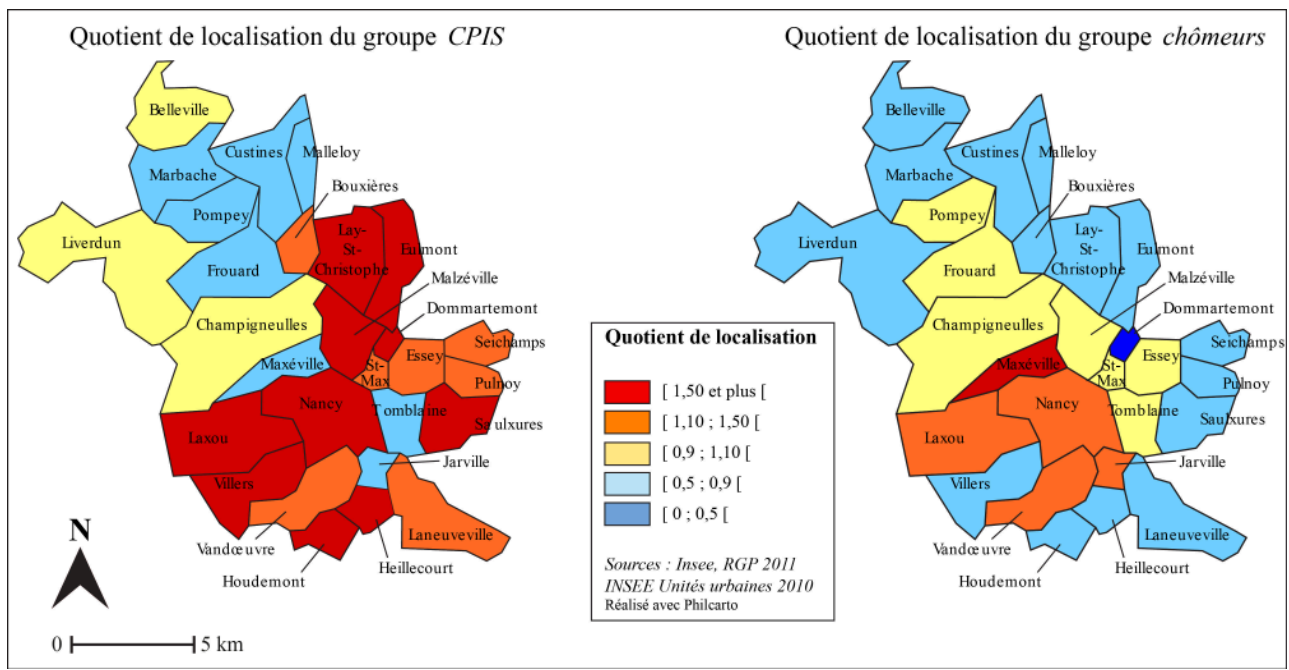

\section{Homogénéisation dans la répartition de la catégorie employés - ouvriers : un lissage spatial qui se confirme}

En 2006, la région Lorraine a connu un renversement socioéconomique important : les employés $(33,4 \%)$ dépassent désormais les ouvriers $(29,9 \%)$ dans le total des emplois salariés (INSEE, populations légales 2006). Pour autant, ces derniers représentent encore trois salariés sur dix et demeurent la deuxième catégorie la mieux représentée. Ce renversement est imputable à la forte poussée de l'emploi tertiaire, mais aussi au recul non négligeable des ouvriers qualifiés, en nombre comme en proportion (Thirion et Schmitt, 2010). Employés et ouvriers constituent la majeure partie de la population active. 
Figure 8: localisation des employés-ouvriers dans les cantons lorrains (2011)

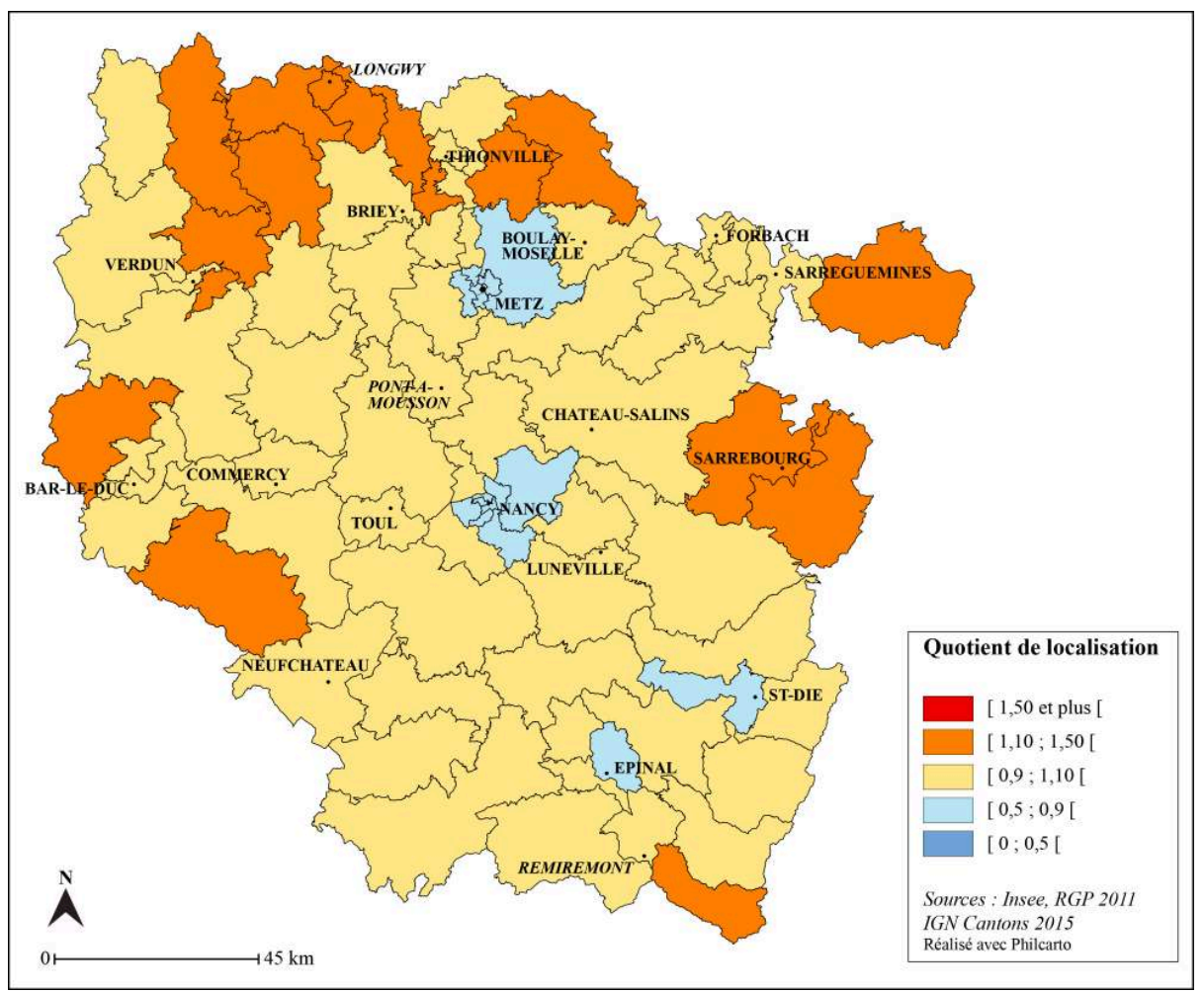

Aussi, les schémas résidentiels des employés-ouvriers se distinguent de ceux étudiés jusque-là. Leur nombre important conduit à une relative homogénéité de leur répartition à l'échelle régionale. Il n'existe plus de foyers de concentration marqués, mais plutôt des secteurs où les représentations sont plus fortes qu'ailleurs : c'est le cas dans les cantons frontaliers des franges septentrionales de la Lorraine, à cheval sur trois départements (la Meuse, la Meurthe-et-Moselle et la Moselle). Ces secteurs sont à la fois des espaces industriels historiques, mais aussi des espaces qui ont profité de la dynamique de l'emploi luxembourgeois. Dans les autres cas, les poches de surreprésentation sont situées sur les marges de la Lorraine (Sarrebourg à l'est et Pays de Bitche au nord-est, Barrois à l'ouest ${ }^{7}$ ), et le long de la frontière (navetteurs vers le Grand-Duché). Les cartographies valorisent en négatif les espaces où le groupe employés-ouvriers est moins représenté. Il s'agit notamment des espaces de concentration des cadres, particulièrement dans les cantons de la périphérie de Metz et de Nancy, mais également dans les cantons des deux principales villes vosgiennes: Épinal et Saint-Dié.

Une exploitation complémentaire des données - en séparant les deux groupes confirme cette première lecture : les ouvriers et les employés ne présentent pas ou peu de secteurs où les représentations sont très fortes, mais ils sont relativement absents dans les cantons périphériques messins et nancéiens. La concentration des employés dans les cantons du nord est bien observable, tandis qu'ils sont sous-représentés dans les cantons des bassins industriels anciens («Moselle-Est » et bassin de Saint-Dié). Les ouvriers sont quant à eux peu présents dans les cantons du Sillon Lorrain. 
Figure 9 : localisation des employés-ouvriers dans les cantons lorrains (1999)

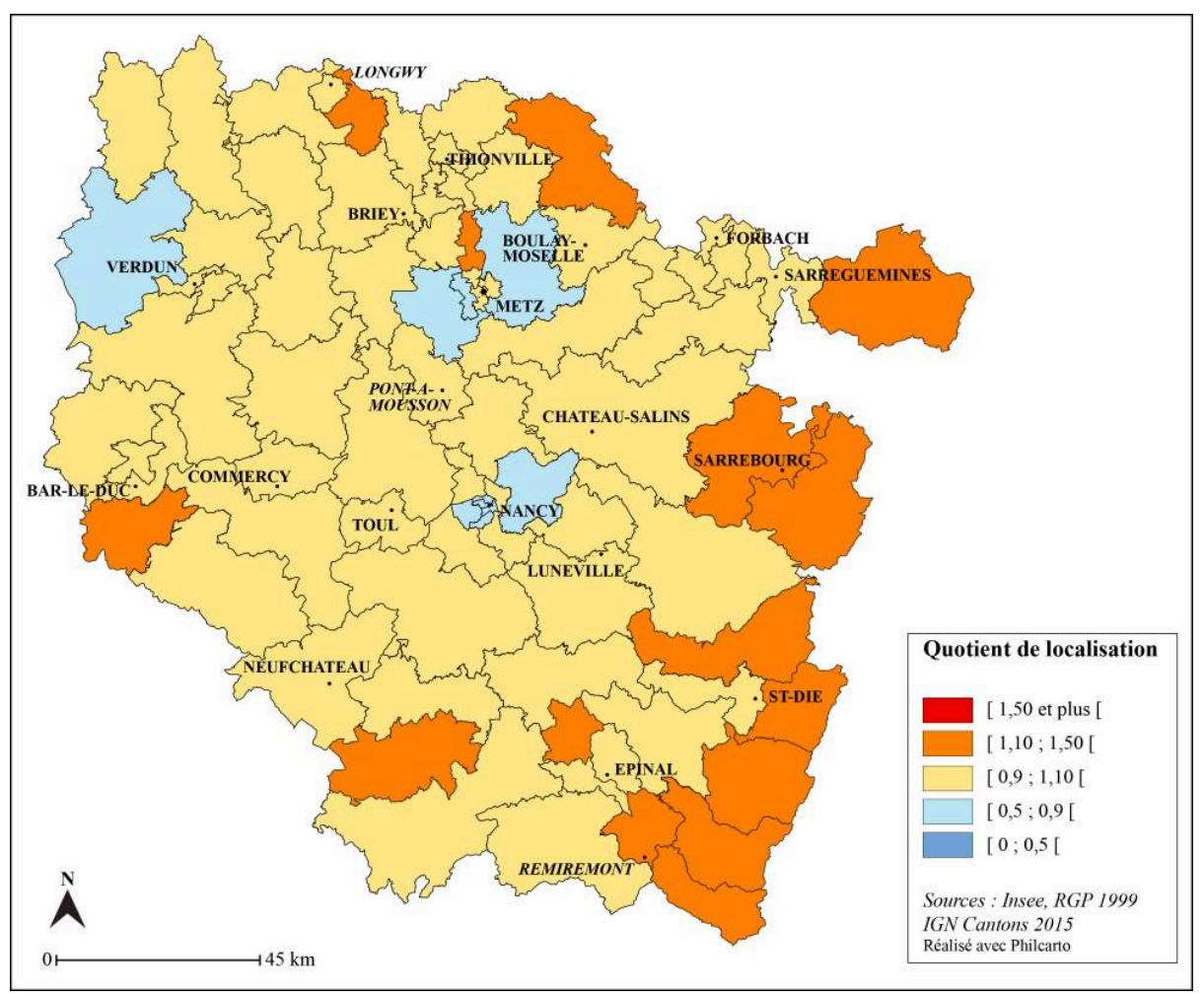

Lors de la décennie 1999-2009, les changements de spatialité résidentielle des employés-ouvriers se sont surtout opérés dans le Massif vosgien et dans les cantons du nord-lorrain. Dans le premier secteur, la surreprésentation observable en 1999 s'est atténuée pour laisser place à des quotients de localisation moyens, et même à de légères sous-représentations à Saint-Dié et Épinal. Dans le nord de la Lorraine, les employés-ouvriers se sont concentrés dans les cantons frontaliers, sauf dans ceux de Thionville et de Yutz (au nord, frontalier du Luxembourg), où, nous l'avons vu, les cadres y sont très présents.

Pour mieux percevoir le différentiel de localisation résidentielle, nous avons choisi de réduire l'analyse à l'aire urbaine de Metz, toujours en utilisant la maille communale (figure 10). Les communes où sont localisés les cadres sont bien distinctes de celles qui abritent les employés-ouvriers. Les communes de la première couronne de Metz montrent de fortes surreprésentations en cadres (carte de droite) et regroupent peu d'employés et d'ouvriers (carte de gauche). Il s'agit essentiellement de communes résidentielles dont une partie est située sur les coteaux de la cuesta de Moselle. Inversement, des communes de banlieue éloignée regroupent peu de cadres mais concentrent les employés-ouvriers. Au nord de Metz, Woippy, qui concentre une part importante de population au chômage et des quartiers de grands ensembles, constitue une exception : les cadres y sont sous-représentés. Il en va de même avec les communes de la vallée sidérurgique de l'Orne et de certaines communes du Saulnois au sud-est de l'aire urbaine. Ce focus sur l'aire messine permet de confirmer les spécialisations communales entre les différentes catégories de population étudiées. Les employésouvriers sont finalement rejetés dans les périphéries plus lointaines, tandis que le nord de l'aire urbaine, contigüe de celle de Thionville, profite de sa bonne connexion aux axes de communication pour être en partie sous influence luxembourgeoise (Diop, 
2011). Plus au sud, la concurrence messine et surtout l'effet-distance atténuent cette influence.

Figure 10 : comparaison des catégories employés-ouvriers et CPIS dans l'aire urbaine de Metz (2011)

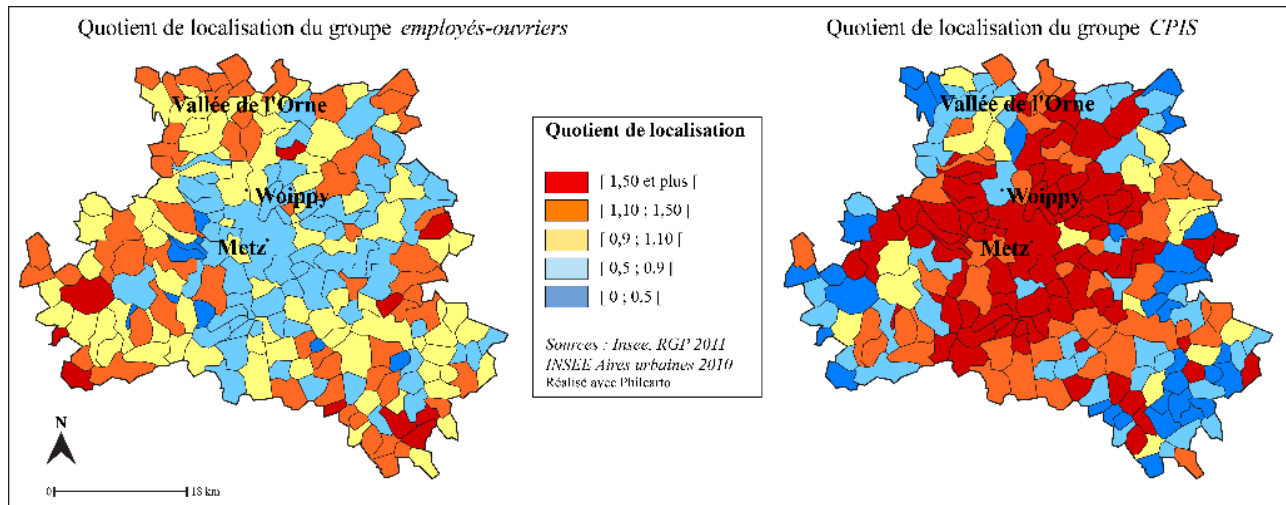

\section{Conclusion}

Les schémas résidentiels observés sont très divers et sont la conséquence d'une triple conjoncture. Pour certains, ils sont liés à la géographie et à l'histoire des lieux (localisation des services rares comme l'université, des bassins ou vallées industrielles). Pour d'autres, ce sont des processus de mobilité accrue qui conduisent des populations à s'installer en périphérie plus ou moins lointaine des agglomérations (nouveaux modes de vie, coût plus faible du foncier), ou dans les centres-villes (aménités urbaines, facilités de déplacement avec les transports publics, etc.). Enfin, la tertiarisation et la montée en qualification de la main d'œuvre, processus encore inachevés en Lorraine, ont montré la place que ces populations occupent au sein d'une région qui lisse ses profils socio-économiques, perd l'image voire le cliché industriel qui se sont longtemps attachés à sa représentation. Cette étude révèle les éléments qui ont conduit à une polarisation de certaines populations, et à l'éviction d'autres. La métropolisation joue un rôle moteur dans l'explication de ces phénomènes, particulièrement dans le Sillon Lorrain.

Ainsi, deux constats se dessinent en Lorraine. D'une part, l'ancienne région confirme des phénomènes socio-spatiaux observables dans les autres régions françaises, comme par exemple dans la région Provence-Alpes-Côte-d'Azur (Fusco et Scarella, 2011). Cela se traduit d'abord par la forte présence des cadres dans les villes-centre et leurs périphéries : c'est particulièrement le cas à Nancy et Metz, où nous avons observé une plus grande concentration des cadres dans les espaces centraux pour la première, et une diffusion plus importante dans le périurbain pour la seconde. En une décennie (1999-2009), les espaces de concentration des cadres se sont étendus sur les cantons périphériques de Metz et de Nancy. Ensuite, les employés-ouvriers présentent une répartition homogène dans le territoire lorrain, sans concentration observable, ni de véritable évolution sur dix ans. Enfin, les chômeurs sont quant à eux localisés dans les principaux pôles et dans les bassins industriels, et sont faiblement sous-représentés ailleurs. L'opposition entre les cadres et les chômeurs se traduit donc dans l'espace par 
une localisation en miroir, ce qui s'observe également à l'échelle de l'unité urbaine nancéienne.

D'autre part, la Lorraine s'individualise par des schémas originaux. En effet, le territoire est dominé par deux villes qui polarisent la population, mais une partie du territoire demeure également sous l'influence grandissante du pôle luxembourgeois. Ainsi, les employés-ouvriers présentent des surreprésentations dans les cantons situés le long de la frontière. Luxembourg et, dans une moindre mesure le nouveau pôle de développement d'Esch-Belval (Del Biondo, 2009), magnétisent ces populations. Par ailleurs, les traces du passé industriel et de son déclin apparaissent encore dans les spatialités de la démographie lorraine : la Moselle et le Massif vosgien sont marqués par des surreprésentations en populations de chômeurs, même si le nombre de cantons concernés a diminué entre 1999 et 2009.

$\mathrm{Au}$ sud du Sillon Lorrain, Épinal est loin de profiter de ces deux dynamiques (métropolitaine et frontalière). La préfecture des Vosges s'illustre seulement comme un pôle départemental sans toutefois jouer le relais de Nancy.

Les disparités socioéconomiques entre population active occupée et population vulnérable mise à l'écart des processus productifs se retranscrivent donc dans le Sillon Lorrain par une forte dichotomie entre les périphéries urbaines les plus proches et les marges métropolitaines où ont tendance à s'installer les processus de paupérisation. Ceci pose d'une part la question de l'équité territoriale entre les espaces dynamiques, économiquement favorisés et hétérogènes du Sillon Lorrain et de la frontière - ce qui leur permet de mieux résister aux crises - et, d'autre part, celle de l'accès au travail des chômeurs, ainsi que du coût à supporter en cas de retour à l'emploi, notamment en matière de mobilité. Une partie de la réponse se situe peut-être dans le choix de résider dans les pôles urbains secondaires qui sont aussi, dans une autre mesure, des pourvoyeurs d'emplois, et demeurent connectés aux grands pôles via le rail notamment, en particulier avec les systèmes de transport express régional (TER).

Néanmoins, l'analyse ne doit pas se réduire à une opposition radicale et réductrice qui rappelle les modèles centre-périphérie (Charmes, 2014). En faisant varier les fenêtres d'observation, nous avons vu qu'il existe des schémas d'opposition à l'échelle des aires urbaines (entre pôle urbain central, communes suburbaines et communes périurbaines) et intra-urbaines, tout comme il y a des espaces aux caractéristiques hétérogènes.

L'attractivité du Luxembourg sur le Nord lorrain crée une situation atypique. Le dynamisme exogène qui embrasse les cantons septentrionaux de la Lorraine a permis d'atténuer partiellement les difficultés liées à la désindustrialisation par les nombreux emplois offerts de l'autre côté de la frontière et ainsi d'infléchir favorablement les statistiques démographiques. Elle interpelle à propos du coût à supporter pour les collectivités : coût financier pour entretenir et améliorer les infrastructures de transport et les services à la population, mais aussi coûts socioéconomiques (effet d'ombre sur l'emploi local) et environnemental (augmentation des migrations pendulaires). La dépendance d'une partie du territoire lorrain face au Grand-Duché interroge le devenir de la Lorraine, dans le contexte de la réforme territoriale ayant conduit à la fusion régionale.

53 En définitive, la méthode des quotients de localisation permet de mettre en lumière les schémas résidentiels des populations-cibles afin de réaliser une première critique des spécialisations en associant des images globales (cadre régional) à des échelles infrarégionales plus fines, jusqu'à se focaliser sur Metz et Nancy. Le quotient de 
localisation a l'avantage de localiser les sous- et surreprésentations des groupes, ce qui s'avère plus pertinent que les indices de concentration ou d'agrégation qui ont tendance à considérer les unités spatiales indépendamment les unes des autres (Apparicio, 2000). Si d'autres indices auraient pu être utilisés (l'indice d'Isard ou l'indice d'entropie par exemple), l'autre intérêt du quotient de localisation est d'avoir été mobilisé sur des études similaires (Fusco et Scarella, 2011; Diop, 2011), ce qui peut introduire une dimension comparative à l'analyse. La méthodologie n'offre toutefois qu'une interprétation parmi d'autres des phénomènes de métropolisation sur le territoire lorrain. En effet, la mobilité joue un rôle central dans ce processus, en parallèle aux logiques foncières et immobilières. Afin de mieux comprendre les enjeux et les conséquences complexes de la métropolisation sur un territoire, il est alors nécessaire de croiser les différentes approches.

\section{BIBLIOGRAPHY}

Apparicio P., 2000, «Les indices de ségrégation résidentielle : un outil intégré dans un système d'information géographique », Cybergeo, article 134. Url : http://cybergeo.revues.org/12063

Ascher F., 2003, « Métropolisation », in J. Lévy J. et M. Lussault (dir.), Dictionnaire de la géographie et de l'espace des sociétés, Paris, pp. 612-615.

Auburtin E., 2014, « Le système de représentations lotharingien », Revue de géographie historique, $\mathrm{n}^{\circ} 4$, mai 2014, en ligne, consulté le 16 février 2016. Url : http://rgh.univ-lorraine.fr/articles/ view/42/Le_systeme_de_representations_lotharingien

Bassand M., 1997, Métropolisation et inégalités sociales, Lausanne, PPUR, 245 p.

Béhar D., Estebe P. et Vanier M., 2011, « Pôles métropolitains : du faire territoire au faire politique », Métropolitiques, 18 mai 2011. Url : http://www.metropolitiques.eu/Polesmetropolitains-du-faire.html

Berrard P.-Y., Debard P, 2011, « Nord lorrain : rebond démographique tiré par la dynamique luxembourgeoise ", Économie Lorraine, INSEE, n²57, 8p.

Bischoff J., Calzada, C., 2010, «Crises et territoires - La Lorraine : une des régions qui ont le plus souffert de la crise », Économie Lorraine, INSEE, n²17, 10 p.

Brun J., Rhein C. (dir.), 1994, La Ségrégation dans la ville. Concepts et mesures, Paris, L'Harmattan, 258 p.

Brutel C., Levy D., 2011, « Le nouveau zonage en aires urbaines de 2010 », INSEE Première, $\mathrm{n}^{\circ}$ $1374,4 \mathrm{p}$.

Calzada C., Cocher C., 2009, « Nouveaux regards sur la métropolisation », Économie Lorraine, INSEE, n¹99-200, en ligne, consulté le 30 mai 2016. Url : http://www.insee.fr/fr/themes/document.asp? reg_id=17\&ref_id=15751

Charmes E., 2014, « Une France contre l'autre ? », La vie des idées, 5 novembre 2014, en ligne, consulté le 16 février 2016. Url : http://www.laviedesidees.fr/Une-France-contre-l-autre.html 
Charmes E., 2011, La ville émiettée : essai sur la clubbisation de la vie urbaine, Paris, PUF, 288 p.

Debrard P., 2008, « La concentration de l'emploi dans le sillon mosellan », Économie Lorraine, INSEE, $n^{\circ} 142$, septembre, 8 p., consulté le 16 février 2016. Url : http://www.insee.fr/fr/ insee_regions/lor/themes/el/el142/el142.pdf

Del Biondo L., 2009, « La conversion des friches sidérurgiques à la frontière francoluxembourgeoise : un enjeu transfrontalier ", L'information géographique, 3/2009 (vol. 73), p. 46-64.

Di Méo G., 2010, « La métropolisation : une clé de lecture de l'organisation contemporaine des espaces géographiques ", L'Information géographique, Vol. 74, pp. 23-38.

Diop L., 2011, « Métropolisation transfrontalière et spécialisation sociale à Luxembourg ", L’Espace géographique, 2011/4, Tome 40, pp. 289-304.

Dodier R., 2004, « Migrations des cadres : entre métropolisation et diffusion », Bulletin de l'Association de géographes français, vol. 81, n² 2, pp. 182-191.

Donzelot J., 2004, « La ville à trois vitesses : relégation, périurbanisation, gentrification », Esprit, n³-4, mars-avril, pp. 14-39, consulté le 16 février 2016. Url : http://www.esprit.presse.fr/ archive/review/article.php?code $=7903$

Dumont G.-F., 2006, L'attractivité des métropoles moyennes en France, Paris, DIACT, 72 p.

Ehrlacher F., 2012, Le transport en Lorraine : les chiffres-clés, Metz, ORT2L, 44 p.

Estèbe P., 2008, Gouverner la ville mobile, Paris, PUF, 76 p.

Frecaut R. (dir.), 1983, Géographie de la Lorraine, Nancy, PUN, Metz, Ed. Serpenoise, 636 p.

Fusco G., Scarella F., 2011, « Métropolisation et ségrégation sociospatiale », L'Espace géographique, 2011/4, Tome 40, pp. 319-336.

Gaschet, F. et Lacour, C. (dir.) (2008), Métropolisation et ségrégation, Pessac, PUB, 316 p.

Ghorra-Gobin, C. (2010) « De la métropolisation : un nouveau paradigme ?», Quaderni, 73, consulté le 22 février 2016. Url : http://quaderni.revues.org/442

Gingembre J., Husson J.-P., 2014, « La naissance d'un Sillon Lorrain élargi : une réponse fonctionnelle et politique », in J. El Gammal, Régions, courants, transferts : histoire et politique, Paris, Riveneuve Éd., p. 195-216.

Girard V., « Des classes populaires (encore) mobilisées ? Sociabilité et engagements municipaux dans une commune périurbaine. ", Espaces et sociétés 1/2014 (n 156-157), p. 109-124.

Julien P., 2002, « Onze fonctions pour qualifier les grandes villes », INSEE Première, $\mathrm{n}^{\circ}$ 840, 4 p., consulté le 16 février 2016. Url : http://www.insee.fr/fr/themes/document.asp?

reg_id=0\&ref_id=IP840

Lacour C., Puissant S., 1999, La métropolisation : croissance, diversités, fractures, Paris, Anthropos, 192 p.

Leroy S., 2000, « Sémantiques de la métropolisation », L'espace géographique, n²9/1, pp. 78-86.

Lussault M., 2003, « Ségrégation », in J. Lévy J. et M. Lussault (dir.), Dictionnaire de la géographie et de l'espace des sociétés, Paris, Belin, pp. 830-832. Url : http://cybergeo.revues.org/26427

Paulet J.-P., 2010, La France : villes et systèmes urbains, Paris, Armand Colin, 222 p.

Perrin E., Rousier N., 2002, « Métropolisation, emploi et politiques locales une relecture de quinze années de recherche en France », Revue d'Économie Régionale \& Urbaine, 5/ 2002, pp. 707-728. 
Piralla S., 2012, « Val de Fensch : ouvert sur ses voisins, le territoire bénéficie depuis 2006 d'un net rebond », Insee Analyses Lorraine, INSEE, n4, 4 p. Url : http://www.insee.fr/fr/themes/ document.asp?reg_id=17\&ref_id=21631

Serre, O., « Sillon Lorrain : un pôle métropolitain articulé autour de Nancy et de Metz », Économie Lorraine, INSEE, $n^{\circ} 313,8 \mathrm{p}$.

Thirion B., Schmitt A., 2010, « L'emploi en Lorraine », Économie Lorraine, INSEE, n² 207, 6 p.

Van Puymbroeck C., Reynard R., 2010, « Répartition géographique des emplois », INSEE Première, $\mathrm{n}^{\circ}$ 1278, 4 p., consulté le 16 février 2016. Url : http://www.insee.fr/fr/themes/document.asp?

reg_id=0\&ref_id=ip1278

Veltz P., 2005, Mondialisation, villes et territoires : l'économie d'archipel, Paris, PUF, 288 p.

\section{NOTES}

1. Depuis le $1^{\mathrm{er}}$ janvier 2016, la Lorraine a été intégrée dans la nouvelle région « Grand Est » avec l'Alsace et la Champagne-Ardenne.

2. Délégation interministérielle à l'aménagement et à la compétitivité des territoires, intégrée depuis 2014 au sein du Commissariat Général à l'Égalité des Territoires.

3. En France, selon les lois du 16 décembre 2010 et du 27 janvier 2014, le pôle métropolitain est un syndicat mixte regroupant des établissements publics de coopération intercommunale à fiscalité propre formant un ensemble de plus de 300000 habitants et dont un des membres compte plus de 100000 habitants. L'intérêt de cette nouvelle structure est double : c'est un syndicat fermé (écartant notamment la région et le département) et la continuité territoriale n'est pas obligatoire, ce qui invite à admettre des sortes d'archipel, des organisations réticulaires, en mosaïque, bref, des formes assez inédites d'approche urbaine.

4. La Grande Région est un espace transfrontalier regroupant le Luxembourg, la Lorraine, la Rhénanie-Palatinat, la Sarre et la Wallonie. Dotée d'une gouvernance rendue complexe par l'hétérogénéité de ses membres, cette « eurorégion » forme un espace de projet aux prérogatives floues. Pourtant, les problématiques qui l'intéressent (onze millions d'habitants, première région européenne en matière de flux domicile-travail) font de cet espace un enjeu majeur pour le développement de ses entités membres et de l'Union Européenne.

5. En 2011, 22232 cadres occupant un emploi résidaient dans les seules communes de Nancy et de Metz, soit près de $20 \%$ du total régional (INSEE).

6. En 2009, la zone d'emploi de Saint-Dié était en Lorraine la plus durement touchée par le chômage (Thirion et Schmitt, 2010).

7. Se reporter à la figure 1 .

\section{ABSTRACTS}

Urban growth, metropolitan process and increasing mobility flows have changed the ways of life in cities. These processes produce spatial differences between the residential patterns of the target populations. 
This paper focuses on the emergence of residential segregation into the functional urban areas of the Lorraine region, in the north-eastern France. The analyses are based on location quotients applied to the cantons.

These results show marked spatial differences across the residential patterns of the target populations, particularly into the urban corridor of Nancy and Metz. The results also show the attractiveness of the highly specialized metropolis of Luxembourg and the city network ("Sillon Lorrain"), as well as the influence of the industrial legacy.

La métropolisation et la mobilité croissante des individus ont bouleversé les façons de vivre et de pratiquer les aires urbaines. C'est le cas en Lorraine, territoire structuré par sa spécificité géographique : d'une part une organisation urbaine polycentrique articulée autour de Nancy, de Metz, et, dans une moindre mesure, de Thionville et d'Épinal (ces quatre villes formant le pôle métropolitain du Sillon Lorrain) ; d'autre part, des espaces frontaliers dynamiques placés sous l'influence croissante du Luxembourg.

Ce travail interroge les relations entre métropolisation régionale, logiques réticulaires et spécialisation résidentielle. Il s'appuie sur la méthode des quotients de localisation et sur une application cartographique multiscalaire employant les contours des nouveaux cantons et des communes. Les résultats révèlent les segmentations spatiales entre les schémas résidentiels des populations-cibles, ainsi que leur évolution depuis la fin des années 1990. Ils soulignent les héritages liés au legs industriel, à l'influence du couloir mosellan mais aussi les mutations récentes liées à l'attractivité luxembourgeoise.

Städtisches Wachstum, Metropolisierung und Mobilität haben die urbane Lebenweise transformieren. Diese Prozesse erzeugen räumliche Disparitäten zwischenWohnmustern der Ziel populationen. Dieses Papier konzentriert sich auf Räumliche Trennung in die funktionalen städtischen Gebieten der Lothringen.

Das methodische Vorgehen für die Analysen basieren auf Standortquotienten der Kantone und Kommunen. Diese Ergebnisse zeigen deutliche Räumliche Differenzierung in den Wohnstrukturen der Zielpopulationen insbesondere im Stadtgebiet Nancy-Metz.

Die Ergebnisse zeigen auch die Attraktivität von Luxemburger Arbeitsmarkt im Lothringen und die Folgen der Deindustrialisierung.

\section{INDEX}

Keywords: city network, location quotient, metropolitan process, Sillon Lorrain, socio-spatial specialization

Mots-clés: métropolisation, quotient de localisation, réseau de villes, Sillon Lorrain, spécialisation résidentielle

Schlüsselwörter. Metropolisierung, Räumliche Spezialisierung, Sillon Lorrain, Städtnetz, Standortquotienten

\section{AUTHOR}

\section{JULIEN GINGEMBRE}

Doctorant - Département de Géographie - LOTERR - Université de Lorraine - 23, boulevard Albert Ier BP 339754015 Nancy CEDEX - julien.gingembre@mailoo.org 